\title{
Support functions for convex domains of finite type
}

\author{
Klas Diederich $^{1}$, John Erik Fornæss ${ }^{2}$ \\ 1 Mathematik, Universität Wuppertal, Gausstr. 20, D-42097 Wuppertal, Germany \\ 2 Department of Mathematics, University of Michigan, East Hall, Ann Arbor, MI 48109- \\ 1109, USA
}

Received April 22, 1998

\section{Introduction}

During the last years much progress has been made towards a better understanding of the complex geometry and analysis of bounded linearly convex domains in $\mathbb{C}^{n}$ with $\mathcal{C}^{\infty}$-smooth boundaries, in particular of those, which are of finite 1-type (in the sense of d'Angelo).

On the geometric side J. Bruna, A. Nagel and S. Wainger clarified 1988 in [4], what the correct notion of pseudoballs and a corresponding pseudometric on convex smooth hypersurfaces of finite type should be. J.McNeal showed in [11], that the d'Angelo 1-type of such domains is at each boundary point realized by a complex line inside the holomorphic tangent space to the boundary at that point. This investigation was continued in [1] and in [15], where it was, finally shown, that the complete Catlin multitype of such hypersurfaces is realized by the orders of contact of linear subspaces of the holomorphic tangent space with the boundary.

A fundamental step on the analytic side was done by in [12] and in [13], which together give quite precise descriptions of the boundary behavior of the Bergman kernel function and the mapping behavior of the Bergman kernel on such domains.

A different type of analytic question, namely the quantitative behavior of the $\bar{\partial}$-equation (in other norms than $L^{2}$-Sobolev), has, except for the case $n=2$ (see [10]), until recently been treated only for a few classes of special convex domains as examples. In [14] the (almost) exact Hölder continuity of solutions to the $\bar{\partial}$-equation for bounded $\bar{\partial}$-closed $(0,1)$-forms was obtained on complex pseudoellepsoids. This was generalized in [2] to a larger class of convex domains of finite type, which are in some sense nevertheless quite 
similar to complex ellipsoids. Later, the exact Hölder continuity properties of such solutions for real pseudoellipsoids were shown in [8]. The reason, for studying this question on real ellipsoids was the following:

A difficulty which arises in the construction of $\bar{\partial}$-solving Cauchy-Fantappié kernels on convex domains $D \subset \subset \mathbb{C}^{n}$ of finite type consists in the fact, that the complex tangent spaces to the boundary realize, as mentioned above, the correct orders of contact in the complex sense, but it may happen, that inside a complex line $l$ tangent to $\partial D$ at a given point $\zeta$ different real lines through 0 might have different orders of contact with $\partial D$. More precisely, there might be exactly one real line inside $l$, which has a strictly larger order of contact than all the other real lines through 0 in $l$. This causes serious difficulties for the estimates if one wants to use the tangent spaces as support hypersurfaces for constructing Cauchy-Fantappié kernels (the same difficulties also appear with respect to some other analytic problems; see, for instance, [3], where exactly this difficulty was avoided by making the additional hypothesis:

There is a constant $\gamma$, such that for each $\zeta \in \partial D$, each unit vector $v \in T_{\zeta}^{10} \partial D$ and each $t>0$ sufficiently small the estimate

$$
\gamma^{-1} r(\zeta+t v) \leq r(\zeta+t i v) \leq \gamma r(z+t v)
$$

holds.

Real pseudoellipsoids are the most simple convex domains of finite type for which this dangerous non-homogeneity of the order of contact of real lines inside a fixed complex tangential line may happen. In [8] they were considered as a test case and new complex support hypersurfaces depending smoothly on the boundary point were constructed which have in all real directions inside a complex tangential direction at any boundary point constant orders of contact, thus enforcing in some sense the hypothesis (1.1) to hold.

The difficulty, that the holomorphic tangent spaces do in general not give the correct supporting functions for convex domains has, recently, been avoided by A. Cumenge who announced in [5] a construction of a $\bar{\partial}$-solving Berndtsson-Andersson kernel on such convex domains by using $\frac{K_{D}(z, w)}{K(w, w)}$ as an approximate peak function on $D$, where $K_{D}$ is the Bergman kernel of $D$. In order to estimate this kernel, the estimates from [12] are used which, in turn, heavily rely on a good knowledge of the $\bar{\partial}$-Neumann problem on such domains.

In this article we will construct $\mathcal{C}^{\infty}$-families of supporting hypersurfaces with optimal orders of contact also in all real directions for arbitrary bounded convex domains with $\mathcal{C}^{\infty}$-boundary of finite type as a useful tool for analytic applications. The basic idea of the technique was, in fact, used for the first time in a different context in [7]. The construction of $\bar{\partial}$ solving integral 
kernels with best possible Hölder estimates using these families of peak functions will be contained in the forthcoming article [6].

The first-named author would like to thank J. McNeal for many conversations on the subject during which, in fact, such optimal supporting hypersurfaces were constructed for arbitrary fixed points on the boundary of the domains considered here (by a different technique), [9]. We also would like to thank B. Fischer for helpful conversations on the subject.

This work was done, while the first author was visiting the Department of Mathematics of the University of Michigan. He would like to thank this institution and, in particular, its complex analysis group for the support and hospitality given to him.

\section{The set-up and the main result}

Let $D \subset \subset \mathbb{C}^{n}$ be a linearly convex domain of finite type $\hat{m}$ with $\mathcal{C}^{\infty}$-smooth boundary and $\zeta_{0} \in \partial D$ an arbitrary point. We denote for $\zeta \in \partial D$ by $n_{\zeta}$ the unit outer normal vector to $\partial D$ at $\zeta$. Then there is a $\mathcal{C}^{\infty}$ family of linear coordinate changes $\left\{l_{\zeta}(z): \zeta \in \partial D\right\}$ composed of a translation and a unitary transformation, such that, for each $\zeta \in \partial D, l_{\zeta}(\zeta)=0$ and $n_{\zeta}$ is turned by $l_{\zeta}$ into the vector $(1,0, \ldots, 0)$. In particular, $T_{\zeta}^{10} \partial D$ becomes in the new coordinates $z=l_{\zeta}(w)$ associated to $\zeta$ just $\left\{z_{1}=0\right\}$. If we write $\partial D$ in these coordinates locally as a graph over its real tangent plane at 0 , given now by $\left\{\operatorname{Re} z_{1}=0\right\}$, we get as a defining function $r_{\zeta}(z)$ for $\partial D$ near $\zeta$ a (uniquely determined) function of the form

$$
\begin{aligned}
r_{\zeta}(z) & =\operatorname{Re} z_{1}+\hat{R}_{\zeta}\left(\operatorname{Im} z_{1}, z^{\prime}\right) \\
& =\operatorname{Re} z_{1}+P_{\zeta}\left(z^{\prime}\right)+R_{\zeta}\left(\operatorname{Im} z_{1}, z^{\prime}\right)
\end{aligned}
$$

where $z^{\prime}=\left(z_{2}, \ldots, z_{n}\right), \hat{R}_{\zeta}(0)=0, d \hat{R}_{\zeta}(0)=0$, and $P_{\zeta}\left(z^{\prime}\right)$ consists of all terms up to total order $\hat{m}$ of the Taylor series of $\hat{R}_{\zeta}$. Hence, $R_{\zeta}$ satisfies the estimates

$$
\left|R_{\zeta}\left(y_{1}, z^{\prime}\right)\right| \leq C\left(y_{1}^{2}+\left|y_{1}\right|\left|z^{\prime}\right|+\left|z^{\prime}\right|^{\hat{m}+1}\right)
$$

for all $\left(y_{1}, z^{\prime}\right)$ close to 0 with a constant $C$ uniform in $\zeta \in \partial D$. More explicitly, we have

$$
P_{\zeta}\left(z^{\prime}\right)=\sum_{j=2}^{\hat{m}} P_{\zeta, j}\left(z^{\prime}\right)
$$

with

$$
P_{\zeta, j}\left(z^{\prime}\right)=\sum_{\alpha+\beta=j} \frac{1}{\alpha ! \beta !} \frac{\partial^{j} r_{\zeta}}{\partial z^{\prime \alpha} \partial \bar{z}^{\prime \beta}}(0) z^{\prime \alpha \bar{z}^{\prime \beta}}
$$




$$
=\sum_{\alpha+\beta=j} a_{\alpha \bar{\beta}}(\zeta) z^{\prime \alpha} \bar{z}^{\prime \beta}
$$

Remark 2.1 Notice, that this expression is invariant under unitary coordinate changes in the $z^{\prime}$-subspace and that all functions $r_{\zeta}, \hat{R}_{\zeta}, P_{\zeta}, R_{\zeta}$ are $\mathcal{C}^{\infty}$ in $(z, \zeta)$ and each $r_{\zeta}$ is convex.

In order to formulate the estimates for the constructed support functions for $D$ precisely and, in fact, also for their proof, we will have to consider intersections of $D$ with transverse 2-dimensional affine subspaces. For this we introduce for any $\zeta \in \partial D$ and any unit vector $v \in T_{\zeta}^{10} \partial D$ the affine space

$$
A_{\zeta, v}:=\left\{z: z=\zeta+w_{1} n_{\zeta}+w_{2} v \text { with } w=\left(w_{1}, w_{2}\right) \in \mathbb{C}^{2}\right\}
$$

and the 2-dimensional convex domain $D_{\zeta, v}:=D \cap A_{\zeta}$. We think of $n_{\zeta}$ and $v$ as being expressed in the coordinates associated to $\zeta$. So, $\zeta=0$, $n_{\zeta}=(1,0 \ldots, 0)$ and $v=\left(0, v_{2}, \ldots, v_{n}\right)$. We may consider $D_{\zeta, v}$ as a domain in $\mathbb{C}^{2}$. It has in the coordinates $\left(w_{1}, w_{2}\right)$ in a neighborhood $W$ of 0 in $\mathbb{C}^{2}$ (independent of $\zeta \in \partial D$ ) the following form

$$
\begin{aligned}
D_{\zeta, v} \cap W:= & \left\{w=\left(w_{1}, w_{2}\right) \in W:\right. \\
& \left.r_{\zeta, v}(w):=r_{\zeta}\left(w_{1} n_{\zeta}+w_{2} v\right)<0\right\}
\end{aligned}
$$

Hence, putting

$$
P_{\zeta, v}(w):=P_{\zeta}\left(w_{2} v\right) \text { and } R_{\zeta, v}\left(\operatorname{Im} w_{1}, w_{2}\right):=R_{\zeta}\left(\operatorname{Im}\left(w_{1} n_{\zeta}\right), w_{2} v\right)
$$

one has

$$
D_{\zeta, v}=\left\{w \in W: \operatorname{Re} w_{1}+P_{\zeta, v}\left(w_{2}\right)+R_{\zeta, v}\left(\operatorname{Im} w_{1}, w_{2}\right)<0\right\}
$$

where $P_{\zeta, v}$ is of the form $P_{\zeta, v}\left(w_{2}\right)=\sum_{j=2}^{\hat{m}} P_{\zeta, v}^{(j)}\left(w_{2}\right)$ with

$$
P_{\zeta, v}^{(j)}(w)=\sum_{k+l=j} a_{k \bar{l}}^{(j)}(\zeta, v) w^{k} \bar{w}^{l}
$$

where the coefficients $a_{k \bar{l}}^{(j)}(\zeta, v)$ are $\mathcal{C}^{\infty}$ in $(\zeta, v) \in \partial D \times \partial B_{n-1}(0 ; 1)$.

We will need the following

Definition 2.2 For any polynomial $P(z)=\sum_{j=0}^{N} \sum_{\alpha+\beta=j} a_{\alpha \bar{\beta}} z^{\alpha} \bar{z}^{\beta}$ on any $\mathbb{C}^{k}$ we define the norm $\|P\|$ to be

$$
\|P\|:=\sum_{j=0}^{N} \sum_{\alpha+\beta=j}\left|a_{\alpha \bar{\beta}}\right|
$$


It also will be convenient to fix the following notation

$$
\sigma_{j}:= \begin{cases}1 & \text { for } j \equiv 0 \bmod 4 \\ -1 & \text { for } j \equiv 2 \bmod 4 \\ 0 & \text { otherwise }\end{cases}
$$

In this article we will show

Theorem 2.3 If in the situation as described above numbers $M, K>0$ have been chosen sufficiently large, then for each sufficiently small $\varepsilon>0$ the function $\hat{S}(z, \zeta) \in \mathcal{C}^{\infty}\left(\mathbb{C}^{n} \times \partial D\right)$, holomorphic in $z$ for each $\zeta \in \partial D$, for which $S_{\zeta}(z):=\hat{S} \circ l_{\zeta}^{-1}(z)$ is (in these new coordinates associated to $\zeta$ ) equal to

$$
S_{\zeta}(z)=3 z_{1}+K z_{1}^{2}-\varepsilon \sum_{j=2}^{\hat{m}} M^{2^{j}} \sigma_{j} \sum_{\substack{|\alpha|=j \\ \alpha=\left(\alpha_{2}, \ldots, \alpha_{n}\right)}} \frac{1}{\alpha !} \frac{\partial^{j} r_{\zeta}}{\partial z^{\prime \alpha}}(0) z^{\prime \alpha}
$$

satisfies the following estimate with a constant $\hat{c}>0$ not depending on $(\zeta, v):$ if we put $S_{\zeta, v}(w):=S_{\zeta}\left(w_{1} n_{\zeta}+w_{2} v\right)$ for $\zeta \in \partial D$ and each unit vector $v \in T_{\zeta}^{10} \partial D$, then

$$
\operatorname{Re} S_{\zeta, v}(w) \leq \frac{\operatorname{Re} w_{1}}{2}-\frac{K}{2}\left(\operatorname{Im} w_{1}\right)^{2}-\epsilon \hat{c} \sum_{j=2}^{\hat{m}}\left\|P_{\zeta, v}^{(j)}\right\|\left|w_{2}\right|^{j}
$$

holds for all $w \in D_{\zeta, v} \cap B_{2}(0 ; \hat{R})$ with a radius $\hat{R}$ independent of $\zeta, v$.

In order to make it clear, what this Theorem means in particular, we formulate the following direct consequence of it:

Corollary 2.4 Define the function $S(z, \zeta)$ on $\mathbb{C}^{n} \times \partial D$ as in Theorem (2.3), take a point $\zeta \in \partial D$ and a unit vector $v \in T_{\zeta}^{10} \partial D$. Let $p \leq \hat{m}$ be the 1-type of the domain $D_{\zeta, v}$ at 0 ( $p$ is the (complex) order of contact of the complex line $\zeta+t v$ with $\partial D$ at $\zeta$ ). Then there is a radius $\hat{R}>0$, not depending on $(\zeta, v)$ and a constant $c>0$, such that the estimate

$$
\operatorname{Re} S(z, \zeta) \leq-c|z-\zeta|^{p}
$$

holds for all $z \in D \cap B(\zeta, \hat{R})$ of the form $z=\zeta+w_{1} n_{\zeta}+w_{2} v$.

Remark 2.5 a) Because of the semicontinuity of the d'Angelo 1-type for convex domains of finite type under small perturbations, our proof shows, that the following more general fact is true:

Let $r$ be a convex $\mathcal{C}^{\infty}$-defining function for a convex bounded domain $D$ with $\mathcal{C}^{\infty}$-smooth boundary of finite type $\hat{m}$. Then there exists a $\eta_{0}>0$ small enough, such that there is a $\mathcal{C}^{\infty}$-function $S(z, \zeta)$, holomorphic in $z$, 
on $\mathbb{C}^{n} \times\left\{\zeta:-\eta_{0} \leq r(\zeta) \leq \eta_{0}\right\}$, which satisfies, for a (suitable) choice of a $\mathcal{C}^{\infty}$-family of coordinate transformations $l_{\zeta}$ with $l_{\zeta}(\zeta)=0$ and turning the exterior normal unit vector $n_{\zeta}$ to $\partial D(r(\zeta)):=\left\{z \in \mathbb{C}^{n}: r(z)=r(\zeta)\right\}$ at $\zeta$ into the vector $(1,0, \ldots, 0) \in \mathbb{C}^{n}$, for sufficiently large constants $K, M$ and sufficiently small $\varepsilon, \hat{R}$, the identity (2.9) and the estimate (2.10) with respect to the domain $D(r(\zeta))$ at $\zeta$ and with the same $\hat{m}$ as in the Theorem. b) It follows directly from our proof of Theorem 2.3, that, instead of using the family of defining functions $r_{\zeta}$ from (2.1) in (2.10), we also could use the family $r_{\zeta}(w):=r \circ l_{\zeta}^{-1}(w)$ as long as $r$ is a $\mathcal{C}^{\infty}$ defining function of $D$ convex on a neighborhood of $\bar{D}$ and such that $|r| \equiv \frac{1}{2}$ on $\partial D$. Formula (2.10) remains valid.

\section{An important Lemma on homogeneous convex polynomials in $\mathbb{C}$}

In this section we will formulate a Main Lemma on homogeneous convex polynomials in one complex variables which will be the basis of the proof of Theorem 2.1.

Lemma 3.1 (Main Lemma) Let $j \geq 2$ be an integer. Then there is an $\varepsilon_{0}(j)>$ 0 , such that for any homogeneous convex polynomial

$$
P_{j}(z)=\sum_{k+l=j} a_{k \bar{l}} z^{k \bar{z}^{l}}
$$

in one complex variable $z$ and any $0<\varepsilon \leq \varepsilon_{0}(j)$ the following inequality holds:

$$
P_{j}(z)+\sigma_{j} \varepsilon \operatorname{Re}\left(a_{j 0} z^{j}\right) \geq \frac{\varepsilon}{2^{j+1}}\left\|P_{j}\right\||z|^{j} \forall z \in \mathbb{C}
$$

with $\sigma_{j}$ as in (2.8).

Remark 3.2 Notice, that $j$ automatically is even.

For the proof of this Lemma we will need the following fact, which we will show first:

Lemma 3.3 Let $P(z)=P(x, y) \neq 0, z=x+i y$, be a homogeneous convex polynomial of degree $j \geq 2$ in the complex plane. Suppose that there is $a z_{0} \neq 0$, such that $P\left(z_{0}\right)=0$. Then $P$ has the form

$$
P(z)=(2 \operatorname{Re}(a z))^{j}
$$

with a suitable $a \in \mathbb{C} \backslash\{0\}$. 
Proof. Notice, that the polynomial $Q(z):=P\left(\frac{z}{z_{0}}\right)$ again is $\neq 0$, convex and homogeneous of degree $j$, since we just made a linear coordinate change. Now we have $Q(1)=0$. We write $Q=Q(x, y)$ in real coordinates. Then we have

$$
Q(x, y)=\sum_{k=0}^{j} b_{k} x^{k} y^{j-k}
$$

However, since $Q(1,0)=0$, there is a $0 \leq s<j$ such that $a_{k}=0$ for all $k>s$. We choose $s$ minimal with this property. Then we have

$$
Q(x, y)=\sum_{k=0}^{s} b_{k} x^{k} y^{j-k}
$$

For any $\delta>0$ the polynomial $Q_{\delta}(x, y):=\delta^{s} Q\left(\frac{x}{\delta}, y\right)$ is again convex and, obviously, $Q_{0}(x, y)=\lim _{\delta \rightarrow 0} Q_{\delta}(x, y)=b_{s} x^{s} y^{j-s}$. In particular, $Q_{0}$ must be convex. This, however, only can be the case for $s=0$, meaning, that $Q(x, y)=a_{0} y^{s}$. From this the claim follows immediately.

We now come to the

Proof of Lemma (3.3). We introduce the following notation:

$$
\mathcal{C}:=\left\{P_{j}: \text { convex, homogeneous of degree } j,\left\|P_{j}\right\|=1\right\}
$$

and assume, that there is no $\varepsilon_{0}(j)>0$, such that (3.1) holds. Then there is a sequence $\left(P_{j}^{(n)}\right)_{n=1}^{\infty} \subset \mathcal{C}$, such that

$$
P_{j}^{(n)}+\frac{1}{n} \sigma_{j} \operatorname{Re}\left(a_{j 0} z^{j}\right)<\frac{1}{2 n}|z|^{j} \text { for some } z \neq 0
$$

We may suppose, that $P_{j}^{(n)} \rightarrow P_{j} \in \mathcal{C}$. There are two possible cases:

$1^{s t}$ case: There is a $c>0$ such that $P_{j}(z) \geq c$ for all $|z|=1$. This, obviously, is a contradiction to (3.3) for large $n$.

$2^{\text {nd }}$ case: There is a $z$ with $|z|=1$, such that $P_{j}(z)=0$. Then, according to Lemma $3.3, P_{j}$ has the form

$$
P_{j}(z)=(a z+\overline{a z})^{j}
$$

with $a \neq 0$. We put $a=t e^{i \psi}$ and $z=r e^{i \theta}$ and calculate

$$
(a z+\overline{a z})^{j}+\varepsilon \sigma_{j} \operatorname{Re}\left(a^{j} z^{j}\right)=t^{j}(\cos (\psi+\theta))^{j}+\varepsilon t^{j} \sigma_{j} \cos (j(\theta+\psi))
$$

Since $j$ is even, we just have to make sure, that $\sigma_{j} \cos (j(\theta+\psi))=1$, whenever $\cos (\theta+\psi)=0$. Since this is exactly the case if $\theta+\psi=k \frac{\pi}{2}$ for $k$ odd, our choice of $\sigma_{j}$ as in (2.8) is good.

The following characterization of convexity in $\mathbb{C}$ can easily be checked: 
Lemma 3.4 A real-valued $\mathcal{C}^{2}$-function $\lambda$ on an open set $U \subset \mathbb{C}$ is convex on $U$ if and only if

$$
\Delta_{c} \lambda(z):=\frac{\partial^{2} \lambda}{\partial z \partial \bar{z}}(z)-\left|\frac{\partial^{2} \lambda(z)}{\partial z^{2}}\right| \geq 0 \forall z \in U
$$

We also will have to consider certain homogeneous polynomials of degree $j \geq 2$ which are almost, but not quite convex. For them we need the

Lemma 3.5 There is a number $\delta_{j}>0$, such that the following holds: If $P_{j}(z)=\sum_{l+k=j} a_{l \bar{k}} z^{l} \bar{z}^{k}$ is a real-valued polynomial on $\mathbb{C}$ which is homogeneous of degree $j$ and which is almost convex in the sense, that there is a $0<\delta<\delta_{j}$ such that

$$
\Delta_{c} P_{j} \geq-\delta\left\|P_{j}\right\||z|^{j-2}
$$

then the inequality

$$
\sigma_{j} \operatorname{Re}\left(a_{j 0} z^{j}\right) \geq \delta\left\|P_{j}\right\|\left|z_{j}\right|
$$

holds for all $z \in \mathbb{C}$ for which

$$
P_{j}(z) \leq \delta\left\|P_{j}\right\||z|^{j}
$$

Proof. We show this indirectly assuming, that there is no such $\delta_{j}$. Then we can find a sequence $P_{j}^{(n)}$ of real-valued homogeneous polynomials of degree $j$, such that $\left\|P_{j}^{(n)}\right\|=1$,

$$
\Delta_{c} P_{j}^{(n)} \geq-\frac{1}{n}|z|^{j-2}
$$

but still there are points $z_{n} \in \mathbb{C},\left|z_{n}\right|=1$, such that $P_{j}^{(n)}\left(z_{n}\right) \leq \frac{1}{n}$, but, nevertheless,

$$
\sigma_{j} \operatorname{Re}\left(a_{j 0}^{(n)} z_{n}^{j}\right)<\frac{1}{n}
$$

We may suppose, that $P_{j}^{(n)} \rightarrow P_{j}$ and $z_{n} \rightarrow z_{0}$. Then, because of (3.4) $P_{j}$ has to be convex, $\left\|P_{j}\right\|=1, P_{j}\left(z_{0}\right) \leq 0$, hence $P_{j}\left(z_{0}\right)=0$ and $\sigma_{j} \operatorname{Re}\left(a_{j 0}^{(0)}\right) \leq 0$. This contradicts Lemma 3.1. * 


\section{Basic estimates on convex functions in $\mathbb{C}$}

Let $\rho_{0} \neq 0$ be a $\mathcal{C}^{\infty}$ convex function in a neighborhood of $\bar{\Delta}=\bar{\Delta}(0 ; \hat{R}) \subset \mathbb{C}$ such that $\rho(0)=0, d \rho(0)=0$. Suppose that the Taylor expansion of $\rho_{0}$ at 0 has the form

$$
\rho_{0}(z)=P_{m}(z)+R_{0}(z)
$$

with a homogeneous polynomial $P_{m} \neq 0$ of degree $m$ and a $\mathcal{C}^{\infty}$-function $R$ such that $\left|R_{0}(z)\right| \leq C|z|^{m+1}$. Because of the convexity of $\rho_{0}$ we have $m=2 r$.

We want to consider in this section the family $\mathcal{F}=\mathcal{F}\left(\rho_{0}\right)$ of all convex functions $\rho$ which are small perturbations of $\rho_{0}$ in the $\mathcal{C}^{2 r+1}$-norm on $\bar{\Delta}$ and such that $\rho(0)=0$ and $d \rho(0)=0$. We can write each such $\rho$ in the form

$$
\rho(z)=\sum_{j=2}^{2 r} P_{j}(z)+R(z)
$$

with polynomials $P_{j}$ homogeneous of degree $j$ and $|R(z)| \leq C|z|^{2 r+1}, C$ independent of $\rho \in \mathcal{F}$. For any numbers $0 \leq R^{\prime} \leq R^{\prime \prime}$ we write $A_{R^{\prime}, R^{\prime \prime}}:=$ $\left\{z: R^{\prime} \leq|z| \leq R^{\prime \prime}\right\}$. We may suppose that the neighborhood $\mathcal{F}$ has been chosen so small that for any $\rho \in \mathcal{F}$ there is a radius $0 \leq R_{2 r-1}<R_{2 r}:=\hat{R}$ such that

$$
\left\|P_{2 r}\right\||z|^{2 r} \geq \max _{2 \leq j<2 r}\left\|P_{j}\right\||z|^{j} \forall z \in A_{R_{2 r-1}, R_{2 r}}
$$

(Notice, that by choosing the neighborhood $\mathcal{F}$ smaller and smaller, we can push $R_{2 r-1}$ arbitrarily close to 0 .)

For each such $\rho$, we choose inductively radii $0 \leq R_{2} \leq \cdots \leq R_{2 r-1} \leq$ $R_{2 r}$ in the following way: if for some $k, 2<k \leq 2 r-1$, the radii $R_{k}, R_{k+1}, \ldots, R_{2 r}$ have already been chosen, then we define $R_{k-1}$ to be the minimum of all radii $0 \leq \tilde{R} \leq R_{k}$ such that

$$
\left\|P_{k}\right\||z|^{k} \geq \max _{2 \leq j<k}\left\|P_{j}\right\||z|^{j} \forall z \in A_{\tilde{R}, R_{k}}
$$

We put $A_{k}:=A_{R_{k-1}, R_{k}}$. Notice, however, that this inequality might not be possible. In such a case we put $R_{k-1}:=R_{k}$. Furthermore, we put $R_{1}:=0$. We note, that we have because of this definition of the $R_{k}$

Lemma 4.1 Choose for a $\rho \in \mathcal{F}$ the radii $0=R_{1} \leq \cdots \leq R_{2 r}$ as above. Then we have for all $2 \leq k \leq 2 r$ and all $z \in \AA_{k}$ the estimate

$$
\left\|P_{k}\right\||z|^{k}>\max _{j \neq k}\left\|P_{j}\right\||z|^{j}
$$


Next, we show, that for any $\rho \in \mathcal{F}$ all those homogeneous parts $P_{k}$ are almost convex, for which the annulus $A_{k}$ is relatively large. More precisely, we have

Lemma 4.2 There is for all $\delta>0$ a number $L_{\delta}>0$ and an arbitrarily small neighborhood $\mathcal{F}$ of $\rho_{0}$, such that for any $\rho \in \mathcal{F}$ and any $k, 2 \leq k \leq 2 r$, for which the radii $R_{k-1}, R_{k}$ corresponding to $\rho$ satisfy $\frac{R_{k}}{R_{k-1}} \geq L_{\delta}$, one has

$$
\Delta_{c}\left(P_{k}\right)(z) \geq-\delta\left\|P_{k}\right\||z|^{k-2} \forall z
$$

Proof. We observe at first, that for a $k$ for which $R_{k}>0$, but $R_{k-1}=0$, the following holds true: $P_{j}=0$ for all $2 \leq j<k$ and $P_{k}$ is convex. Hence (4.5) is trivially satisfied for such $k$.

Next, we notice that for some universal constant $C>0$ and any homogeneous polynomial $P_{j}$ of degree $2 \leq j \leq 2 r$ we have by the triangle inequality

$$
\left|\Delta_{c} P_{j}(z)\right| \leq C\left\|P_{j}\right\||z|^{j-2} \forall z
$$

Hence, we get for any $\rho \in \mathcal{F}$ represented in the form (4.2) and any $2 \leq k \leq$ $2 r$ because of the convexity of $\rho$

$$
\Delta_{c}\left(P_{k}\right) \geq-C \sum_{j \neq k}\left\|P_{j}\right\||z|^{j-2}-\left|\Delta_{c}(-R)\right|
$$

We may suppose, that $\left|\Delta_{c}(-R)\right| \leq C|z|^{2 r-1}$ for any $\rho \in \mathcal{F}$ and we define for any $2 \leq k \leq 2 r$ such that $R_{k-1} \neq 0$

$$
\hat{R}_{k}:=\sqrt{R_{k-1} R_{k}}
$$

Put for such a $k$ the ratio $\frac{R_{k}}{R_{k-1}}=: L$ and choose $j \neq k$. We consider the $1^{\text {st }}$ case: $j>k$

Then we get

$$
\begin{aligned}
\left\|P_{j}\right\| \hat{R}_{k}^{j} & =\left\|P_{j}\right\| R_{k-1}^{\frac{j}{2}} R_{k}^{\frac{j}{2}} \\
& =\left\|P_{j}\right\| R_{k}^{j} \frac{R_{k-1}^{j / 2} R_{k}^{j / 2}}{R_{k}^{j}} \\
& \leq\left\|P_{k}\right\| R_{k}^{k} \frac{R_{k-1}^{j / 2}}{R_{k}^{j / 2}} \text { because of the definition of } R_{k} \\
& =\left\|P_{k}\right\| \hat{R}_{k}^{k} \frac{R_{k}^{k / 2}}{R_{k-1}^{k / 2}} \cdot \frac{R_{k-1}^{j / 2}}{R_{k}^{j / 2}} \\
& \leq\left\|P_{k}\right\| \hat{R}_{k}^{k} L^{(k-j) / 2}
\end{aligned}
$$


Together with (4.6) we get in this first case

$$
\left|\Delta_{c} P_{j}(z)\right| \leq C\left\|P_{k}\right\| \hat{R}_{k}^{k-2} \frac{1}{L^{(j-k) / 2}}
$$

Next we have to consider the

$2^{\text {nd }}$ case: $j<k$

By the same chain of estimates we get here

$$
\left|\Delta_{c} P_{j}(z)\right| \leq C\left\|P_{k}\right\| \hat{R}_{k}^{k-2} \frac{1}{L^{(k-j) / 2}}
$$

Plugging (4.8) and (4.9) into (4.7) gives

$$
\Delta_{c}\left(P_{k}\right) \geq-C\left\|P_{k}\right\| \hat{R}_{k}^{k-2} \sum_{j \neq k} \frac{1}{L^{|k-j| / 2}}-C \hat{R}_{k}^{2 r-1}
$$

Notice, that, whatever $L>0$ is, the neighborhood $\mathcal{F}$ of $\rho_{0}$ can be chosen so small, that we have

$$
C \hat{R}_{2 r}=C \sqrt{R_{2 r-1} R_{2 r}} \leq \frac{\left\|P_{2 r}\right\|}{L}
$$

for all $\rho \in \mathcal{F}$. Hence, we also have $C \hat{R}_{k} \leq \frac{\left\|P_{2 r}\right\|}{L}$. This gives

$$
C \hat{R}_{k}^{2 r-1} \leq \frac{1}{L}\left\|P_{2 r}\right\| \hat{R}_{k}^{2 r-2} \leq \frac{1}{L}\left\|P_{k}\right\| \hat{R}_{k}^{k-2}
$$

We put this into (4.10) and see, that the Lemma follows by just choosing $L$ large enough.

Now we have to take into account also those terms $P_{l}$, for which the corresponding annuli are very small or even just circles. We show

Lemma 4.3 Let $\delta>0$ be fixed and choose $L_{\delta}>0$ and the neighborhood $\mathcal{F}$ of $\rho_{0}$ according to Lemma 4.2. Also fix a constant $\hat{C}>0$. Then for any sufficiently large constant $M>0$ the following holds true:

Suppose the pair $(j, k), 2 \leq j<k \leq 2 r$, has the following properties with respect to a function $\rho \in \mathcal{F}$ :

a) $\frac{R_{l}}{R_{l-1}} \leq L_{\delta}$ for all $j<l<k$,

b) $\AA_{j} \neq \emptyset$ and $\AA_{k} \neq \emptyset$.

Then one has

$$
M^{2^{k}}\left\|P_{k}\right\||z|^{k}+M^{2^{j}}\left\|P_{j}\right\||z|^{j}>\hat{C} M^{2^{l}}\left\|P_{l}\right\||z|^{l} \forall j<l<k, \forall z
$$


Proof. We define inductively: $N_{2 r}:=\left\|P_{2 r}\right\|$ and if $N_{l+1}$ has already been defined we put for any $l \geq 2$

$$
N_{l}:=\left\{\begin{array}{l}
N_{l+1} R_{l} \text { if } \stackrel{\circ}{A}_{l}=\emptyset \\
\left\|P_{l}\right\| \text { if } \stackrel{\circ}{A}_{l} \neq \emptyset
\end{array}\right.
$$

We claim, that we then have for any $2 \leq l<2 r$ the equation

$$
N_{l} R_{l}^{l}=N_{l+1} R_{l}^{l+1}
$$

Namely, observe, that this follows from (4.12), if $\AA_{l}=\emptyset$. If $\AA_{l} \neq \emptyset$, let $\lambda$ be the smallest positive integer such that also $\AA_{l+\lambda} \neq \emptyset$. Then we have according to the definition of the radii $R_{i}$ the equation

$$
\left\|P_{l}\right\| R_{l}^{l}=\left\|P_{l+\lambda}\right\| R_{l+\lambda-1}^{l+\lambda}
$$

Since, in this case, $N_{l}=\left\|P_{l}\right\|$ and $N_{l+\lambda}=\left\|P_{l+\lambda}\right\|$ and since, furthermore, $R_{l}=R_{l+1}=\cdots=R_{l+\lambda-1}$, (4.13) follows.

Let, for a given $\rho \in \mathcal{F}$, the pair $(j, k)$ be chosen as in the Lemma. We fix any $j<l<k$ and consider points $z$ with $|z|^{k-l}>\hat{C} \frac{M^{2^{l}}}{M^{2^{k}}} R_{l} \cdots R_{k-1}$. We insert into this $R_{i}=\frac{N_{i}}{N_{i+1}}$ and obtain after cancelations

$$
|z|^{k-l}>\hat{C} \frac{M^{2^{l}}}{M^{2^{k}}} \frac{N_{l}}{N_{k}}
$$

Now, notice, that $N_{k}=\left\|P_{k}\right\|$ and, even if $\AA_{l}=\emptyset$, we still have $\left\|P_{l}\right\| \leq N_{l}$. Putting this into (4.14) and multiplying by $|z|^{l}$, we get

$$
M^{2^{k}}\left\|P_{k}\right\||z|^{k}>\hat{C} M^{2^{l}}\left\|P_{l}\right\||z|^{l}
$$

for all $|z|^{k-l}>N_{l, k}:=\hat{C} \frac{M^{2^{l}}}{M^{2^{k}}} R_{l} \cdots \cdot R_{k-1}$.

On the other hand we consider points $z$ with $|z|^{l-j} \leq \frac{M^{2^{j}}}{\hat{C} M^{2^{l}}} R_{j} \cdots$ $R_{l-1}=\frac{M^{2^{j}}}{\hat{C} M^{2}} \frac{N_{j}}{N_{l}}$. As above we obtain

$$
M^{2^{j}}\left\|P_{j}\right\||z|^{j} \geq \hat{C} M^{2^{l}}\left\|P_{l}\right\||z|^{l}
$$

for all $|z|^{l-j} \leq \hat{N}_{l, j}:=\frac{M^{2^{j}}}{\hat{C} M^{2^{l}}} R_{j} \cdots R_{l-1}$.

We want to show, that for $M$ sufficiently large (independent of the choice of $j, k$ ) we have

$$
N_{k, l}^{\frac{1}{k-l}}<\hat{N}_{j, l}^{\frac{1}{l-j}}
$$


For this we calculate

$$
\begin{aligned}
\frac{\hat{N}_{j, l}^{\frac{1}{l-j}}}{N_{k, l}^{\frac{1}{k-l}}}= & \frac{1}{\hat{C}^{\frac{1}{l-j}+\frac{1}{k-l}}} \cdot M^{\frac{2^{j}-2^{l}}{l-j}-\frac{2^{l}-2^{k}}{k-l}} \\
& \cdot\left(R_{j} \cdot \ldots \cdot R_{l-1}\right)^{\frac{1}{l-j}}\left(R_{l} \cdot \ldots \cdot R_{k-1}\right)^{-\frac{1}{k-l}}
\end{aligned}
$$

and observe that

$$
\left(R_{j} \cdots \cdot R_{l-1}\right)^{\frac{1}{l-j}}\left(R_{l} \cdots \cdot R_{k-1}\right)^{-\frac{1}{k-l}} \geq \frac{R_{j}}{R_{k-1}} \geq \frac{1}{L_{\delta}^{k-1-j}} \geq \frac{1}{L_{\delta}^{2 r}}
$$

because of property a) of $l$ in the Lemma.

Alltogether this implies the Lemma.

In order to get the complete estimate of Theorem 2.3 we still need to include the remainder term $R(z)$ of any $\rho \in \mathcal{F}$ into our estimates. For this we use the following fact:

Lemma 4.4 For $A>0$ sufficiently large one has: for each $\rho=\sum_{j=2}^{2 r} P_{j}+$ $R \in \mathcal{F}$ the function $\rho_{A}:=\sum_{j=2}^{2 r} P_{j}+A|z|^{2 r+1}$ is convex (outside 0 ).

Proof. Notice, that $\Delta_{c}|z|^{2 r+1}=\left(\frac{2 r+1}{2}\right)^{2}|z|^{2 r-1}-\frac{2 r+1}{2} \frac{2 r-1}{2}|z|^{2 r-1}=$ $\left(\frac{2 r+1}{2}\right)|z|^{2 r-1}$ and that for $\rho \in \mathcal{F}$ always $\left|\Delta_{c} R\right| \leq C|z|^{2 r-1}$. Hence, choosing $A$ very large makes the function $\rho_{A}$ convex everywhere.

We call $\mathcal{F}_{A}:=\left\{\rho_{A}: \rho \in \mathcal{F}\right\}$ and apply Lemma 2.1 from [4] to the family $\mathcal{F}_{A}$. We get

Lemma 4.5 There is a universal constant $C_{\hat{m}}$ (notice, that $2 r \leq \hat{m}$ ), such that for all $\rho_{A} \in \mathcal{F}_{A}$

$$
\rho_{A}(z) \geq C_{\hat{m}}\left(\sum_{j=2}^{2 r}\left|P_{j}(z)\right|+A|z|^{2 r+1}\right)
$$

For simplicity of notation, we call for a given $\rho \in \mathcal{F}$ an index $j, 2 \leq j<2 r$, good, if for a given $\delta>0$ and $L_{\delta}$ chosen according to Lemma 4.2 the corresponding annulus $A_{j}$ satisfies $\frac{R_{j}}{R_{j-1}}>L_{\delta}$. Otherwise, $j$ is, of course bad. We get from Lemmas 4.3 and 4.5

Lemma 4.6 Choose $\mathcal{F}, L_{\delta}, M, \hat{C}, A$ as above. Then there is a constant $c>0$ such that for all $\rho \in \mathcal{F}$ and any $\varepsilon>0$

$$
\rho_{A}(z)+\varepsilon \sum_{j=2}^{2 r} M^{2^{j}} \sigma_{j} \operatorname{Re}\left(a_{j 0} z^{j}\right)
$$




$$
\begin{aligned}
& \geq c\left(\sum_{j \text { good }}\left|P_{j}(z)\right|+A|z|^{2 r+1}\right) \\
& \quad+\varepsilon \sum_{j \text { good }} M^{2^{j}}\left(\sigma_{j} \operatorname{Re}\left(a_{j 0} z^{j}\right)-\frac{2 r}{\hat{C}}\left\|P_{j}\right\||z|^{j}\right)
\end{aligned}
$$

Proof. Notice that, according to Lemma 4.3 we can choose for any index $l$, which is bad, good indices $j<l<k$ such that

$$
\begin{aligned}
{M^{2}}^{l} \sigma_{l} \operatorname{Re}\left(a_{l 0} z^{l}\right) & \geq-M^{2^{l}}\left\|P_{l}\right\||z|^{l} \\
& \geq-\frac{1}{\hat{C}}\left(M^{2^{j}}\left\|P_{j}\right\||z|^{j}+M^{2^{k}}\left\|P_{k}\right\||z|^{k}\right)
\end{aligned}
$$

Using this we get

$$
\begin{aligned}
& \rho_{A}(z)+\varepsilon \sum_{j=2}^{2 r} M^{2 j} \sigma_{j} \operatorname{Re}\left(a_{j 0} z^{j}\right) \\
& \geq \text { (Lemma 4.5) } C_{\hat{m}}\left(\sum_{j=2}^{2 r}\left|P_{j}(z)\right|+A|z|^{2 r+1}\right)+\varepsilon \sum_{j=2}^{2 r} M^{2^{j}} \sigma_{j} \operatorname{Re}\left(a_{j 0} z^{j}\right) \\
& \geq C_{\hat{m}}\left(\sum_{j \operatorname{good}}\left|P_{j}(z)\right|+A|z|^{2 r+1}\right)+\varepsilon \sum_{j \operatorname{good}} M^{2^{j}} \sigma_{j} \operatorname{Re}\left(a_{j 0} z^{j}\right) \\
& \quad+\varepsilon \sum_{j \text { bad }} M^{2^{j}} \sigma_{j} \operatorname{Re}\left(a_{j 0} z^{j}\right) \\
& \geq \text { (Lemma 4.3) } C_{\hat{m}}\left(\sum_{j \operatorname{good}}\left|P_{j}(z)\right|+A|z|^{2 r+1}\right) \\
& \quad+\varepsilon \sum_{j \operatorname{good}} M^{2^{j}} \sigma_{j} \operatorname{Re}\left(a_{j 0} z^{j}\right)-\varepsilon \frac{2 r}{\hat{C}} \sum_{j \operatorname{good}} M^{2^{j}}\left\|P_{j}\right\||z|^{j}
\end{aligned}
$$

From this the Lemma follows.

The following Lemma puts together what has been proved in this section:

Lemma 4.7 Let for a given $\mathcal{C}^{\infty}$ convex function $\rho_{0}$ on a neighborhood of $\bar{\Delta}$ a sufficiently small neighborhood $\mathcal{F}$, a fixed number $\delta>0$ smaller than all the $\delta_{j}$ from Lemma 3.5, $L_{\delta}>0$ according to Lemma 4.2 and $A>0$ according to Lemma 4.4 be chosen. If then $\hat{C}>\frac{4 r}{\delta}$ and $M>0$ is sufficiently 
large, then there is a constant $\hat{c}>0$ such that for all $\varepsilon>0$ sufficiently small there is a radius $\hat{R}>0$, such that the following estimate holds:

$$
\rho(z)+\varepsilon \sum_{j=2}^{2 r} M^{2^{j}} \sigma_{j} \operatorname{Re}\left(a_{j 0} z^{j}\right) \geq \varepsilon \hat{c} \sum_{j=2}^{2 r}\left\|P_{j}\right\||z|^{j} \quad \forall|z|<\hat{R}
$$

for all $\rho \in \mathcal{F}$.

Proof. $\rho \in \mathcal{F}$ and choose the radii $R_{j}$ according to (4.4). According to Lemma 4.6 we then have for the corresponding $\rho_{A}$

$$
\begin{aligned}
\rho_{A}(z) & +\varepsilon \sum_{j=2}^{2 r} M^{2^{j}} \sigma_{j} \operatorname{Re}\left(a_{j 0} z^{j}\right) \geq c \sum_{j \text { good }}\left|P_{j}(z)\right| \\
& +\varepsilon \sum_{j \text { good }}\left(M^{2^{j}} \sigma_{j} \operatorname{Re}\left(a_{j 0} z^{j}\right)-\frac{2 r}{\hat{C}}\left\|P_{j}\right\||z|^{j}\right)
\end{aligned}
$$

Now we consider at first for any fixed good $j$ all points $z$, such that

$$
P_{j}(z)<\delta\left\|P_{j}\right\||z|^{j}
$$

We get from Lemma 3.5

$$
\sigma_{j} \operatorname{Re}\left(a_{j 0} z^{j}\right) \geq \delta\left\|P_{j}\right\||z|^{j}
$$

and hence, according to the choice of $\hat{C}$

$$
\varepsilon M^{2^{j}} \sigma_{j} \operatorname{Re}\left(a_{j 0} z^{j}\right)-\frac{2 r \varepsilon}{\hat{C}}\left\|P_{j}\right\||z|^{j} \geq \frac{\varepsilon \delta}{2} M^{2^{j}}\left\|P_{j}\right\||z|^{j}
$$

If, on the other hand, for this $j$ the point $z$ has been chosen such that

$$
P_{j}(z) M \geq \delta\left\|P_{j}\right\||z|^{j}
$$

then we get for $\varepsilon>0$ small enough (depending just on $j, M, \hat{C}, c$ )

$$
c\left|P_{j}(z)\right|+\varepsilon M^{2^{j}} \sigma_{j} \operatorname{Re}\left(a_{j 0} z^{j}\right)-\frac{2 r \varepsilon}{\hat{C}}\left\|P_{j}\right\||z|^{j} \geq \frac{c \delta}{2}\left\|P_{j}\right\||z|^{j}
$$

If $\varepsilon>0$ is small enough, we have $\frac{\varepsilon \delta}{2} M^{2^{j}}<\frac{c \delta}{2}$. Putting together (4.19), (4.20) and (4.21) we get

$$
\rho_{A}(z)+\varepsilon \sum_{j=2}^{2 r} M^{2^{j}} \sigma_{j} \operatorname{Re}\left(a_{j 0} z^{j}\right) \geq \frac{\varepsilon \delta}{2} \sum_{j \text { good }} M^{2^{j}}\left\|P_{j}\right\||z|^{j} \quad \forall z
$$


Because of Lemma 4.3 we can even allow in the right side of this inequality also the terms for which $j$ is not good after we divide by $2 r$ (notice, that $\hat{C}>1$ ). We obtain

$$
\rho_{A}(z)+\varepsilon \sum_{j=2}^{2 r} M^{2^{j}} \sigma_{j} \operatorname{Re}\left(a_{j 0} z^{j}\right) \geq \frac{\varepsilon \delta}{4 r} \sum_{j=2}^{2 r} M^{2^{j}}\left\|P_{j}\right\||z|^{j} \forall z
$$

If we now choose a radius $\hat{R}>0$ small enough, we get

$$
A|z|^{2 r+1}+|R(z)|<\frac{\varepsilon \delta}{8 r}\left\|P_{2 r}\right\||z|^{2 r} \forall|z| \leq \hat{R}
$$

Combining this with (4.22) we get with $\hat{c}:=\frac{\delta}{8 r}$

$$
\rho(z)+\varepsilon \sum_{j=2}^{2 r} M^{2^{j}} \sigma_{j} \operatorname{Re}\left(a_{j 0} z^{j}\right) \geq \varepsilon \hat{c} \sum_{j=2}^{2 r}\left\|P_{j}\right\||z|^{j}
$$

for all $|z|<\hat{R}$ and for all $\rho \in \mathcal{F}$. This proves the Lemma.

\section{Inequalities in $\mathbb{C}^{2}$}

We, next, want to draw the necessary consequences from Lemma (4.7) for a class of convex functions on $\mathbb{C}^{2}$ which will, for a given convex domain $D \subset \subset \mathbb{C}^{n}$ with $\mathcal{C}^{\infty}$-boundary and any $\zeta \in \partial D, v \in T_{\zeta}^{10} \partial D$ with $|v|=1$, include the function $r_{\zeta, v}$ as defined in (2.5). We show

Proposition 5.1 Let $\tilde{\rho}_{0}\left(w_{1}, w_{2}\right)=\operatorname{Re} w_{1}+\tilde{R}^{(0)}\left(\operatorname{Im} w_{1}, w_{2}\right)$ be a $\mathcal{C}^{\infty}$ convex function satisfying $\tilde{R}^{(0)}(0)=0, d \tilde{R}^{(0)}(0)=0$ and which is of finite type at 0 in the sense, that it can be written as

$$
\tilde{\rho}_{0}(w)=\operatorname{Re} w_{1}+\tilde{P}^{(0)}\left(w_{2}\right)+\tilde{R}_{1}^{(0)}\left(\operatorname{Im} w_{1}, w_{2}\right)
$$

with a homogeneous polynomial $P^{(0)}\left(w_{2}\right) \neq 0$ of degree say $2 r$ and

$$
\left|\tilde{R}_{1}^{(0)}\left(\operatorname{Im} w_{1}, w_{2}\right)\right| \leq C\left(\left|\operatorname{Im} w_{1}\right|\left|w_{2}\right|+\left|\operatorname{Im} w_{1}\right|^{2}\right)
$$

Fix any integer $\hat{m} \geq 2 r$. Then there is a neighborhood $\hat{\mathcal{F}}$ of $\tilde{\rho}_{0}$ in the $\mathcal{C}^{\infty}$-topology and there are constants $K, M$ (sufficiently large), $\varepsilon, \hat{R}>0$ (sufficiently small) and $\hat{c}>0$ (sufficiently small), such that for all functions from

$$
\begin{aligned}
\tilde{\mathcal{F}}:= & \left\{\tilde{\rho} \in \hat{\mathcal{F}}: \tilde{\rho}(w)=\operatorname{Re} w_{1}+\tilde{R}\left(\operatorname{Im} w_{1}, w_{2}\right) \text { convex },\right. \\
& \tilde{R}(0)=0, d \tilde{R}(0)=0\}
\end{aligned}
$$


the following holds:

If we associate with $\tilde{\rho}$ the function

$$
S(w):=3 w_{1}+K w_{1}^{2}-\varepsilon \sum_{j=2}^{\hat{m}} M^{2^{j}} \sigma_{j} \frac{1}{j !} \frac{\partial^{j} \tilde{\rho}}{\partial w_{2}^{j}}(0) w_{2}^{j}
$$

with $\sigma_{j}$ as in (2.8) then the inequality

$$
\operatorname{Re}(S(w)) \leq \frac{\operatorname{Re} w_{1}}{2}-\frac{K}{2}\left(\operatorname{Im} w_{1}\right)^{2}-\frac{\varepsilon \hat{c}}{2} \sum_{j=2}^{\hat{m}}\left\|\tilde{P}_{j}\right\|\left|w_{2}\right|^{j}
$$

holds on $\{w: \tilde{\rho}(w) \leq 0\} \cap B(0 ; \hat{R})\}$, where

$$
\tilde{P}_{j}\left(w_{2}\right):=\sum_{k+l=j} \frac{1}{k ! l !} \frac{\partial^{j} \tilde{\rho}}{\partial w_{2}^{k} \partial \bar{w}_{2}^{l}}(0) w^{k} \bar{w}^{l}
$$

for $j=2, \ldots, \hat{m}$.

Proof. We use all notations of Sect. 4 and observe, that Lemma 4.7 applies to $\tilde{\rho}\left(0, w_{2}\right)$ for all $\tilde{\rho} \in \tilde{\mathcal{F}}$, if only $\hat{\mathcal{F}}$ has been chosen small enough. Putting $w_{1}=x+i y$, we write $\tilde{\rho} \in \tilde{\mathcal{F}}$ in the form

$$
\tilde{\rho}\left(w_{1}, w_{2}\right)=x+\tilde{\rho}\left(0, w_{2}\right)+y \cdot \tilde{R}_{2}\left(y, w_{2}\right)
$$

We use polar coordinates $w_{2}=t e^{i \theta}$. The convexity of $\tilde{\rho}$ gives, in particular,

$$
\frac{\partial^{2} \tilde{\rho}}{\partial y^{2}} \frac{\partial^{2} \tilde{\rho}}{\partial t^{2}}-\left(\frac{\partial \tilde{R}_{2}}{\partial t}\right)^{2} \geq 0
$$

at points of the form $\left(0, t e^{i \theta}\right)$. Now, observe at first, that

$$
\left|\frac{\partial^{2} \tilde{\rho}}{\partial y^{2}}\left(0, t e^{i \theta}\right)\right| \leq C
$$

for a constant $C>0$ not depending on the choice of $\tilde{\rho} \in \tilde{\mathcal{F}}$. Furthermore, since $\tilde{\rho}\left(0, t e^{i \theta}\right)=\sum_{j=2}^{2 r} \tilde{P}_{j}\left(t e^{i \theta}\right)+\tilde{R}\left(t e^{i \theta}\right)$ with $\tilde{R}\left(w_{2}\right)=O\left(\left|w_{2}\right|^{2 r+1}\right)$ uniformly in $\tilde{\rho} \in \tilde{\mathcal{F}}$, we have

$$
\left|\frac{\partial^{2} \tilde{\rho}}{\partial t^{2}}\left(0, t e^{i \theta}\right)\right| \leq \sum_{j=2}^{2 r}\left\|\tilde{P}_{j}\right\| t^{j-2}+C t^{2 r-1}
$$


with another constant $C>0$ not depending on $\tilde{\rho}$. Putting (5.5) and (5.6) into (5.4) gives after possibly increasing $C$ and observing, that $\left\|\tilde{P}_{2 r}\right\|$ is uniformly bounded away from 0

$$
\left|\frac{\partial \tilde{R}_{2}}{\partial t}\left(0, t e^{i \theta}\right)\right| \leq C \sum_{j=2}^{2 r} \sqrt{\left\|\tilde{P}_{j}\right\|} t^{\frac{j}{2}-1}
$$

Hence, we obtain by integration changing $C$ on the way appropriately

$$
\left|\tilde{R}_{2}\left(0, w_{2}\right)\right| \leq C \sum_{j=2}^{2 r} \sqrt{\left\|\tilde{P}_{j}\right\|}\left|w_{2}\right|^{j / 2} \leq \sqrt{C \sum_{j=2}^{2 r}\left\|\tilde{P}_{j}\right\|\left|w_{2}\right|^{j}}
$$

Now we can estimate the real part of the function $S(w)$ from (5.2) for any given $\tilde{\rho} \in \tilde{\mathcal{F}}$ on $D \cap B(0, \hat{R})$ for sufficiently small $\hat{R}>0$ where

$$
D:=\left\{w \in \mathbb{C}^{2}: \tilde{\rho}(w)<0\right\}
$$

using Lemma 4.7. Namely, we have on $D$

$$
x<-\sum_{j=2}^{2 r} \tilde{P}_{j}\left(w_{2}\right)-\tilde{R}\left(w_{2}\right)-y \tilde{R}_{2}\left(y, w_{2}\right)
$$

Putting this into (5.2) we get

$$
\begin{aligned}
\operatorname{Re}(S(w)) \leq & -|x|-2 \sum_{j=2}^{2 r} \tilde{P}_{j}\left(w_{2}\right)-2 \tilde{R}\left(w_{2}\right)-2 y \tilde{R}_{2}\left(y, w_{2}\right) \\
& +K x^{2}-K y^{2}-\varepsilon \sum_{j=2}^{\hat{m}} \sigma_{j} M^{2^{j}} \operatorname{Re}\left(a_{j 0} w_{2}^{j}\right)
\end{aligned}
$$

where $a_{j 0}$ is the coefficient of the term $w_{2}^{j}$ in $\tilde{P}_{j}$. We split the last sum in this expression into 2 parts, namely, $\sum_{j=2}^{2 r} \ldots$ and $\sum_{j=2 r+1}^{\hat{m}} \ldots$. Obviously the second part is $O\left(\left|w_{2}\right|^{2 r+1}\right)$ uniformly in $\tilde{\rho}$. Furthermore, Lemma 4.7 gives

$$
\begin{aligned}
& -\sum_{j=2}^{2 r} \tilde{P}_{j}\left(w_{2}\right)-\tilde{R}\left(w_{2}\right)-\varepsilon \sum_{j=2}^{2 r} M^{2^{j}} \sigma_{j} \operatorname{Re}\left(a_{j 0} w_{2}^{j}\right) \\
& \leq-\varepsilon \hat{c} \sum_{j=2}^{2 r}\left\|\tilde{P}_{j}\right\|\left|w_{2}\right|^{j} \forall\left|w_{2}\right|<\hat{R}
\end{aligned}
$$


Furthermore, $\left|\tilde{R}_{2}\left(y, w_{2}\right)\right|=O\left(|y|+\left|w_{2}\right|\right)$ uniformly on $\tilde{\mathcal{F}}$. Putting all this together with (5.7) into (5.8), we get for $w \in D \cap B(0, \hat{R})$, after possibly shrinking $\hat{R}$, the estimate

$$
\operatorname{Re} S(w) \leq-\frac{|x|}{2}-\varepsilon \hat{c} \sum_{j=2}^{2 r}\left\|\tilde{P}_{j}\right\|\left|w_{2}\right|^{j}+|y| \sqrt{C \sum_{j=2}^{2 r}\left\|\tilde{P}_{j}\right\|\left|w_{2}\right|^{j}}-\frac{K}{2} y^{2}
$$

Finally, we apply the Peter-Paul-inequality to this and get after choosing $K$ sufficiently large

$$
\operatorname{Re} S(w) \leq-\frac{|x|}{2}-\frac{K}{2} y^{2}-\frac{\varepsilon \hat{c}}{2} \sum_{j=2}^{\hat{m}}\left\|\tilde{P}_{j}\right\|\left|w_{2}\right|^{j}
$$

for all $w \in D \cap B(0, \hat{R})$. This finishes the proof of Proposition 5.1.

Remark 5.2 Notice, that Proposition 5.1 contains Theorem 2.3 for the case of dimension 2 .

\section{Proof of Theorem 2.3}

Let now $D \subset \subset \mathbb{C}^{n}$ be a convex domain with $\mathcal{C}^{\infty}$-smooth boundary $\partial D$ of finite type $\hat{m}$. We use the complete set-up and notations as described in the beginning of Sect. 2 of this article. We fix an arbitrary point $\zeta_{0} \in \partial D$ and a unit vector $v_{0} \in T_{\zeta}^{10} \partial D$. We, then, can apply Proposition 5.1 taking the function $r_{\zeta, v}(w)$ as defined in (2.5) as $\tilde{\rho}_{0}(w)$. For the 1-type $\hat{m}$ of $D$ we have, indeed, $\hat{m} \geq 2 r$, where $2 r$ is the 1-type of the convex domain in $\mathbb{C}^{2}$ defined by $r_{\zeta, v}$. We now choose $\tilde{\mathcal{F}}, K, M, \varepsilon, \hat{R}, \hat{c}$ as given by Proposition 5.1. Then there is a neighborhood $\hat{T}$ of $\left(\zeta_{0}, v_{0}\right)$ in $\partial D \times T_{\zeta}^{10}$, such that for any $(\zeta, v) \in \hat{T}$ the function $r_{\zeta, v}$ as defined by (2.5) belongs to $\tilde{\mathcal{F}}$. Hence, we can write down the function $S_{\zeta, v}(w)$ given by (5.2) for each $(\zeta, v) \in \hat{T}$ and the estimate (5.3) will hold on $D_{\zeta, v} \cap B(0 ; \hat{R})$ (see (2.5)).

Next notice, that there is a relatively open neighborhood $\hat{V}$ of $\zeta_{0}$ in $\partial D$, such that $\hat{V} \times \partial B_{n-1}(0 ; 1)$ as a subset of $\partial D \times T^{10} \partial D$ is covered by a finite number of such neighborhoods $\hat{T}$. Taking the maximal constants $K, M$ and the minimal constants $\varepsilon, \hat{R}, \hat{c}$ used for these finitely many neighborhoods and write down for each $(\zeta, v) \in \hat{V} \times \partial B_{n-1}(0 ; 1)$ the function $S_{\zeta, v}$. Then the $S_{\zeta, v}$ are automatically of the form $S_{\zeta, v}(w)=S_{\zeta}\left(w_{1} n_{\zeta}+w_{2} v\right)$ with $S_{\zeta}$ as in (2.9), and (2.10) holds.

Finally, we can cover $\partial D$ by finitely many of these neighborhoods $\hat{V}$. Taking again $K, M$ maximal and $\varepsilon, \hat{R}, \hat{c}$ minimal, Theorem 2.3 follows. 


\section{References}

1. Boas, H.P., Straube, E.J.: On equality of type and variety type of real hypersurfaces in $\mathbb{C}^{n}$, J. Geom. Analysis 2 (1992), 95-98

2. Bruna, J., Castillo, J. del: Hölder and $L^{p}$-estimates for the $\bar{\partial}$-equation in some convex domains with real-analytic boundary, Math. Ann. 269 (1984), 527-539

3. Bruna, J., Charpentier, P., Dupain, Y.: Zero varieties for the Nevanlinna class in convex domains of finite type in $\mathbb{C}^{n}$, To appear in Adv.Math., 1997

4. Bruna, J., Nagel, A., Wainger, S.: Convex hypersurfaces and Fourier transform, Ann. Math. 127 (1988), 333-365

5. Cumenge, A.: Estimées Lipschitz optimales dans les convexes de type fini, C. R. Acad. Sci. Paris 325 (1997), 1077-180

6. Diederich, K., Fischer, B., Fornæss, J.E.: Hölder estimates on convex domains of finite type, In preparation

7. Diederich, K., Fornæss, J. E.: Strictly pseudohyperbolic domains, manuscripta math. 25 (1978), 263-278

8. Diederich, K., Fornæss, J.E., Wiegerinck, J.: Sharp hölder estimates for $\bar{\partial}$ on ellipsoids, Manuscripta math. 56 (1986), 399-417

9. Diederich, K., McNeal, J.: Supporting hypersurfaces for convex domains of finite type, Unpublished Notes, 1995

10. Fefferman, C., Kohn, J. J.: Hölder estimates on domains of complex dimension two and on three dimensional CR manifolds, Adv. Math. 69 (1988), 233-303

11. McNeal, J.: Convex domains of finite type, J. Functional Anal. 108 (1992), 361-373

12. McNeal, J.: Estimates on the Bergman kernel of convex domains, Adv. Math. 109 (1994), 108-139

13. McNeal, J., Stein, E. M.: Mapping properties of the Bergman projection on convex domains of finite type, Duke Math. J. 73 (1994), 177-199

14. Range, R. M.: On Hölder estimates for $\bar{\partial} u=f$ on weakly pseudoconvex domains, Proceedings of International Conferences, Cortona, Italy 1976-1977, Sc. Norm. Sup. Pisa, pp. 247-267

15. Yu, J.Y.: Multitype of convex domains, Indiana Univ. Math. J. 41 (1992), 837-849 